\title{
Longitudinal Study of Iron Deposition and Volume in the Precentral Gyrus in Patients with Relapse-Remitting Multiple Sclerosis
}

\author{
Silin Du, Chun Zeng, Zhiwei Zhang* \\ The First Affiliated Hospital of Chongqing Medical University, Chongqing, China \\ Email: *zhangzhiweicqmu@163.com
}

How to cite this paper: Du, S.L., Zeng, C. and Zhang, Z.W. (2020) Longitudinal Study of Iron Deposition and Volume in the Precentral Gyrus in Patients with Relapse-Remitting Multiple Sclerosis. Journal of Biosciences and Medicines, 8, 84-95.

https://doi.org/10.4236/jbm.2020.85008

Received: March 21, 2020

Accepted: May 3, 2020

Published: May 6, 2020

Copyright (c) 2020 by author(s) and Scientific Research Publishing Inc. This work is licensed under the Creative Commons Attribution International License (CC BY 4.0).

http://creativecommons.org/licenses/by/4.0/

\begin{abstract}
Objective: To longitudinally assess dynamic changes of iron deposition and volume of the precentral gyrus and its correlation with clinical manifestations of Relapse-Remitting Multiple Sclerosis(RRMS) by using 3D enhanced $\mathrm{T}_{2}^{*}$ weighted angiography(ESWAN). Methods: Thirty RRMS patients and thirty age- and sex-matched healthy controls were recruited and underwent ESWAN and $3 \mathrm{D} \mathrm{T}_{1} \mathrm{WI}$ twice interval of one year with the same parameters. The mean phase values (MPVs) and volumes in precentral gyrus gray matter (PGM) were measured, and change of iron content and its correlation with volume, clinical manifestations were analyzed. Results: Compared with controls, the RRMS had higher iron deposition in both single-time measurements, but the volume decreased. Comparing to the first scan, we found significant difference in MPVs between the two times $(P<0.05)$ in the RRMS, while the volumes had no significant difference. A significant positive correlation was found between the MPVs and the volume (first time $r_{s}=0.764, P<0.05$; second time $r_{s}=0.592$, $P<0.05)$ in RRMS. MPVs was positively correlated with volume $\left(r_{s}=0.582, P\right.$ $<0.05)$. Duration disease was negatively correlated with the second MPVs $\left(r_{s}\right.$ $=-0.399, P<0.05)$, and expended disability status scale (EDSS) was negatively correlated with the volume change $\left(r_{s}=-0.745, P<0.05\right)$. The recurrence rate was negatively correlated with the change of MPVs $\left(r_{s}=-0.367, P<0.05\right)$. Conclusions: With the disease progression, the content of iron in PGM in RRMS patients is increasing, while the volume has no obvious change, suggesting that the iron deposition may precede or develop faster than cerebral atrophy.
\end{abstract}

\section{Keywords}

Relapse-Remitting Multiple Sclerosis, Precentral Gyrus, Iron, Brain Atrophy 


\section{Introduction}

Multiple sclerosis (MS) is an autoimmune-mediated inflammatory demyelinating disease, leading to widespread damage throughout the central nervous system (CNS) [1]. While historically considered a disease of the white matter (WM), MS is now widely understood as having gray matter (GM) as well. Moreover, many studies have investigated a stronger correlation between GM lesions and physical disability, fatigue and cognitive deficits than with lesion burden, suggesting that GM measurements are of great clinical importance [2]. Although iron is an essential component for proper homeostasis in CNS, excessive iron within the deep GM may is associated with both physical disability and cognitive impairment in MS patients [3]. However, the mechanisms of iron accumulation in MS have not been incompletely understood. It may be the case that iron concentrations are secondary consequences stemming from WM injury. In this situation, neuronal loss or axonal transection may cause interruptions within the WM pathways and subsequently trigger neurodegenerative processes and/or alter transport of iron [4]. Recent reports have documented that the levels of iron content were found to be elevated in numerous neurological disorders, such as multiple sclerosis (MS) [5], Parkinson's disease (PD) [6]. Several MRI-based different techniques have been used for studying iron concentration, including $\mathrm{T}_{2}$ hypointensity, $\mathrm{R}_{2}{ }^{*}$ relaxometry, SWI high-pass filtered phase imaging and Quantitative Susceptibility Mapping (QSM). Due to its sensitivity to paramagnetic substances, SWI high-pass filtered phase imaging allows for the indirect characterization of iron deposition, primarily ferritin.

The precentral gyrus is obvious thickness and can be identified easily on three-dimensional (3D) $\mathrm{T}_{1}$ images. Moreover, the precentral gyrus is the location of the primary motor cortex. It works together with other motor areas to plan, initiate and execute movements. The cross-time iron measurements may not be sufficient to determine whether iron content is pathologically changing in individual MS patients. Longitudinal analysis would be more powerful in understanding the iron pathophysiologic processes in MS or may represent a new method of classifying disease severity. Recently a longitudinal study by Walsh AJ et al. [6], it has shown that using different methods $\mathrm{R}_{2}{ }^{*}$ mapping and phase imaging to evaluate iron content in the deep GM between MS patients and control subjects, iron content significantly increases in the deep gray matter of MS patients during two years.

To our knowledge, this is the first study to longitudinally evaluate abnormal iron concentration and the volume in the precentral gyrus GM, using longitudinal 3D-ESWAN over a 2-year period. We propose were to investigate the changes of iron deposition in the precentral gyrus GM of RRMS. Furthermore, we also evaluated whether iron levels in the precentral gyrus GM were associated with precentral gyrus volume, disease duration, EDSS scores of MS patients. 


\section{Materials and Methods}

\subsection{Patient Selection}

Thirty patients with RRMS and 30 age- and sex-matched healthy control (HC) subjects, who were recruited from the First Affiliated Hospital of Chongqing Medical University (Chongqing, China), were studied from Nov 2015 to May 2018 in this study. Each subject was imaged twice, two year apart. RRMS patients were graded according to the expanded disability status scale (EDSS) Disease duration is as measured from the date of the original diagnosis to the second scan and the times of recurrence is as measured from the first time symptom appearing to the second scan. The criteria for inclusion of RRMS patients were based on the following: 1) The diagnosis of RRMS was made according to the 2010 revised McDonald criteria; 2) Age 16 - 70 at baseline; 3) All RRMS patients were treated with glucocorticoid before entering the study and during the follow-up period. The criteria for inclusion of heathy control groups were based on the following: No trauma; No intracranial hemorrhage; No intracranial occupation; Intracranial non-information. Exclusion criteria for all subjects were that they had other neurologic diseases or that they had contraindications to MR imaging. The main characteristic and clinical characteristics of the patients are shown in Table 1.

This study was supported by the institutional review board of Chongqing Medical University and complied with the Health Insurance Portability and Accountability Act. Written informed consent was obtained from all participants at the beginning of the study.

\subsection{MRI Data Acquisitions}

All MR images were performed using a 3.0 Tesla (T) MR scanner (Signa, HD, General Electric [GE] Healthcare, Milwaukee, WI, USA) equipped with an eight-channel phased array head coil. All subjects were subjected to the standard MRI protocols

Table 1. Main characteristic of RRMS patients and control subjects.

\begin{tabular}{|c|c|c|}
\hline & RRMS group & Controls group \\
\hline Number of subjects & 30 & 30 \\
\hline $\operatorname{Sex}(M / F)$ & $12 / 18$ & $12 / 18$ \\
\hline Age scale (year) & $16-69$ & $16-69$ \\
\hline Median age (year) & $48.4 \pm 13.4$ & $45.5 \pm 14.5$ \\
\hline EDSS range (the first scan) & $1-6.5$ & - \\
\hline Mean EDSS (the first scan) & $2.07 \pm 1.71$ & - \\
\hline EDSS range (the $2^{\text {nd }}$ scan) & $0.5-7.5$ & - \\
\hline Mean EDSS (the $2^{\text {nd }}$ scan) & $2.12 \pm 1.69$ & - \\
\hline Disease duration range (year) & $1-13$ & - \\
\hline Mean disease duration (year) & $4.64 \pm 2.9$ & - \\
\hline
\end{tabular}

RRMS: Relapse-Remitting Multiple sclerosis; EDSS: Expanded disability status scale. 
proposed by the international MS group diagnosis center. The parameter of conventional MR imaging are as follows: 1) a conventional axial 2D multi-planar dual fast spin-echo (FSE) proton density (PD) and $\mathrm{T}_{2}$-weighted images (WI): repetition time $[\mathrm{TR}] 2900 \mathrm{~ms}$, echo time $[\mathrm{TE}]_{1} /[\mathrm{TE}]_{2} 25 \mathrm{~ms} / 93 \mathrm{~ms}$, echo train length [ETL] 12, matrix size $256 \times 192 ; 2$ ) an axial T 1 WI:TR $8000 \mathrm{~ms}$, TE $120 \mathrm{~ms}$, inversion time [TI] $2000 \mathrm{~ms}$, ETL 8, matrix size $256 \times 160$; and 3) fluid attenuated inversion recovery (FLAIR): TR $2050 \mathrm{~ms}$, TE $24 \mathrm{~ms}$, TI $750 \mathrm{~ms}$, matrix size $256 \times 256$. These routine sequences were acquired with 5 -mm-thick contiguous sections, no space and a $24 \mathrm{~cm} \times 24 \mathrm{~cm}$ field of view (FOV).

All ESWAN sequences were acquired using a 3D-enhanced $\mathrm{T}_{2}{ }^{*}$ susceptibilityweighted angiography contrast flow compensated (i.e., the gradient moment was null in all three orthogonal directions) multi-echo (eight different TE) gradient echo sequence which was performed with the following parameter: TR $60 \mathrm{~ms}$, effective TE $6 \mathrm{~ms}\left(\mathrm{TE}_{\mathrm{i}} 5.8-54.4 \mathrm{~ms}\right.$, where $\mathrm{TE}_{\mathrm{i}}$ is the effective TE range), FOV 22 $\mathrm{cm} \times 22 \mathrm{~cm}$, slice thickness $2 \mathrm{~mm}$, matrix size $488 \times 320$, bandwidth 31.25 $\mathrm{Hz} /$ pixel and flip angle $0^{\circ}$. We also acquired $3 \mathrm{D} \mathrm{T}_{1}$ spoiled gradient (SPGR) echo images with the following parameters: TE min full, TR $8.3 \mathrm{~ms}$, FOV $24 \mathrm{~cm} \times 24$ $\mathrm{cm}$, flip angle $12^{\circ}$, bandwidth $27.28 \mathrm{~Hz} /$ pixel, matrix size $256 \times 256$, NEX 1, slice thickness $1.0 \mathrm{~mm}$. All scans were oriented parallel to the anterior-posterior commissural (AC-PC) line with 56 to 64 locations on the middle sagittal plane and covered the entire brain parenchyma. The total acquisition time was between 6 and $8 \mathrm{~min}$, depending on the spatial ratio and the number of sections.

\section{Data Processing and Analysis}

\subsection{Data Processing}

Phase images serve as a direct measure of magnetic field variation. Based on the following formulas: $\phi$ (phase) $=-r \Delta \mathrm{B} \mathrm{TE}$ (where $r$ signifies gyromagnetic, $\Delta \mathrm{B}$ is the change in the magnetic field between tissues and TE is the echo time); $\Delta \mathrm{B}=c \mathrm{~V} \Delta x \mathrm{~B}_{0}$ (where $c$ is the iron content, $\mathrm{V}$ is the voxel volume and $\Delta x$ is the variation in molar susceptibility between tissues in the presence of iron) [7]. As the amount of iron in a tissue increases, the phase change between tissues decreases for the same TE level. Therefore, for a given TE level, $c$ and appear to be negatively correlated.

All ESWAN images were post-processed using research software (FuncTool 6.3.1e software, GE Healthcare) on an ADW4.6 workstation (Sun Microsystems, Santa Clara, Calif). Following post-processing, high-pass filtered phase and magnitude images are automatically presented. The phase images of all subjects were copied to the computer for iron concentration quantification by the SPIN software (Signal Processing in Nmr; http://www.mrc.wayne.edu/download). First, high-pass filtered phase images were read by the SPIN software. Observer was allowed to adjust the brightness and contrast of each of the phase images to obtain improved definition of anatomical reference structures and consequently greater precision in measurement, as shown in Figure 1. Second, the regions of interests (ROIs) were magnified the same times for a clearer definition of the 
margins and manually drawn on these images. Thirdly, the means and standard deviations of the signal intensity measurements of the ROIs the MPVs were obtained. To avoid bias because of signal heterogeneity, care was taken to choose the most homogeneous region. To ensure data accuracy, all ROI sections were measured three times with the same method, and the final MPVs were taken as the average phase value of the nine sections. All structural MRI post-processing was performed by an observer who was unaware of identity of the patients and their various diagnoses.

For each participant, The precentral gyrus gray matter were investigated.PGM was acquired including the section with the area in the longest central sulcus and the adjacent superior and inferior area. And then the MPVs were averaged between hemispheres. Next, axial 3D $\mathrm{T}_{1}$ images were transferred to the observer's computer and using the Image $\mathrm{J}$ image analysis software (Image Processing and Analysis in Java; rsb.info.nih.gov/ij/).We evaluated the anatomical location of precentral gyrus, as shown in Figure 2. Firstly, the central sulcus is a prominent
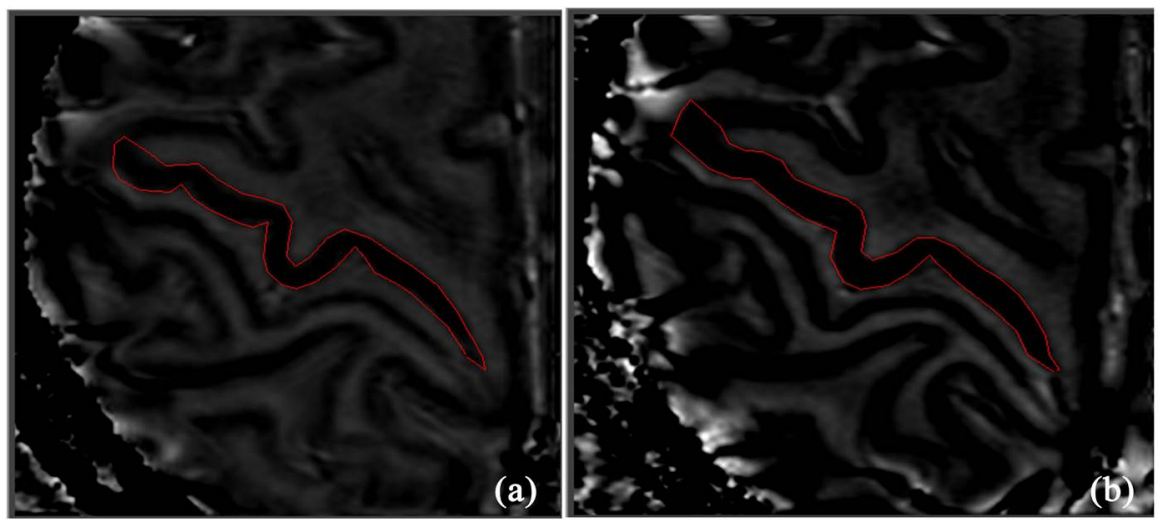

Figure 1. (a) The phase-ESWAN of RRMS patient at the first examination and the contour of the phase value of the precentral gyrus matter was measured; (b) The phaseESWAN of a same RRMS patient at the second examination, compared the first time, the phase value of the precentral gyrus matter was decreased.
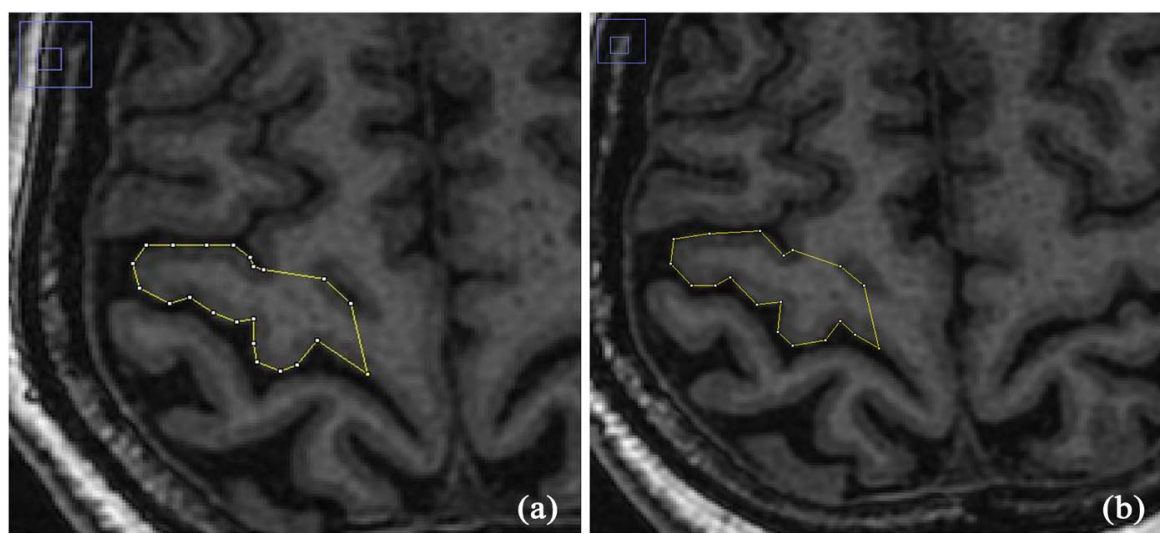

Figure 2. (a) The $\mathrm{T}_{1} \mathrm{WI}$ of RRMS patient at the first examination and the contour of the volume of the precentral gyrus was measured; (b) The $\mathrm{T}_{1} \mathrm{WI}$ of a same RRMS patient at the second examination, compared the first time, the volume of the precentral gyrus was no significant change. 
landmark of the brain, it is the longest sulcus. The precentral gyrus is located on the anterior of the central sulcus. Secondly, we also defined the precise location of the motor hand area (a knob-like structure) to guide identification of the precentral gyrus. Furthermore, the EDSS neurological disability scores of the MS patients were evaluated by other observer who was unaware of the MRI results.

\subsection{Statistical Analysis}

Statistical analysis was performed using the SPSS Statistical package (SPSS for windows, software version 19.0; SPSS, Chicago, IL, USA). The mean and standard deviation (SD) were calculated. Correlations analyses were conducted by using the Spearman's rank correlation coefficient to investigate relations between the MPVs of the PGM, the volume of PGM, disease duration, the EDSS scores and the times of recurrence. In addition, the paired-samples $t$ test was conducted to compare the two-time data to tract if there was a significant change in two year periods, and the student's $t$-test was performed to determine if there was a significant difference the MPVs of the precentral gyrus GM in MS patients compared to those of healthy controls. All tests were two-tailed $(\alpha=0.05)$, and $P$ value of less than 0.05 was considered to indicate a statistically significant difference.

\section{Results}

The mean phase values (MPVs) in the PGM in the RRMS patients in two times were $2033.131 \pm 14.39$ and $2022.65 \pm 17.94$, respectively, and the MPVs in controls were $2050.11 \pm 20.35$ and $2048.83 \pm 19.26$. According to the previous study [8], we observed a negative correlation between the MPVs of the PGM and the post-mortem brain iron concentration of healthy adults. And had significant difference in the RRMS group compared to the controls $(P<0.05)$ in both single-time measurements.

The volumes in the precentral gyrus in RRMS were $2307.82 \pm 109.24\left(\mathrm{~mm}^{3}\right)$ and $2216.82 \pm 118.70\left(\mathrm{~mm}^{3}\right)$, respectively. And the volumes of the controls were $2357.67 \pm 102.45\left(\mathrm{~mm}^{3}\right)$ and $2310.7 \pm 108.29\left(\mathrm{~mm}^{3}\right)$. The volumes of the precentral gyrus in RRMS patients had significant decrease in both single-time compared to healthy controls, and had significant difference $(P<0.05)$. Compared to the first time scan, using the Paired $t$ test, the volume in the second time scan of the PGM there was no obvious change and had no significant difference $(P>$ 0.05).

Spearman's rank correlation coefficient analysis showed that there was a significant negative correlation between the MPVs and the volumes in the PGM in RRMS in the two scans $\left(r_{s}=0.764, P<0.05 ; r_{s}=0.592, P<0.05\right)$. For the thirty RRMS patients, the mean EDSS scores at the baseline were $2.07 \pm 1.71$ (range 1 to 6.5), and the median EDSS score at follow-up was $2.12 \pm 1.69$ (range 0.5 to 7.5), but no correlations was found between the EDSS scores and the MPVs $(P>0.05)$. There was significant positive correlation in RRMS between the precentral gray 
matter MPVs and the volume of precentral gyrus for the first time $\left(r_{s}=0.764, P\right.$ $<0.05)$. The same results were acquired for the second time $\left(r_{s}=0.592, P<\right.$ 0.05). There was positive correlation between the changes of MPVs and the volume of precentral gray matter $\left(r_{s}=0.582, P<0.05\right)$. There was negative correlation between duration disease and the MPVs for the second time $\left(r_{s}=-0.399, P\right.$ $<0.05$ ) (Figure 3 ), but there was no correlation between the change of EDSS and the change of MPVs $(P>0.05)$. However, there was significant negative correlation between the change of EDSS and the change of the volume of precentral gyrus $\left(r_{s}=-0.745, P<0.05\right)$. Significant negative correlation was observed between the recurrence rate and the change of MPVs $\left(r_{s}=-0.367, P<0.05\right)$.

\section{Discussion}

Enhanced $\mathrm{T}_{2}{ }^{*}$-weighted angiography imaging (ESWAN) is a robust and powerful tool, because of its high spatial resolution and susceptibility weighting of the phase image. ESWAN uses the magnetic susceptibility difference between oxygenated and eoxygenated haemoglobin to allow to observe of iron deposition in the basal ganglia region and gray matter [9]. To our knowledge, this is the first study which longitudinally quantifies iron accumulation and evaluates the relationship between the iron deposition and its volume and other clinical characteristics in the PGM in MS patients using ESWAN. In our study, we had four main outcomes. First, we demonstrated that iron deposition exhibited increase with the disease progress RRMS patients in the PGM. Secondly, the volume of the PGM had no obvious change in the following period. Thirdly, there was positive correlation between the changes of MPVs and the volume of precentral gray matter, suggesting that the iron deposition maybe precede on the brain atrophy or the iron deposition developed faster than atrophy, the iron deposition maybe would result in the brain atrophy in someday, but the exact mechanism was not unclear. Fourthly, the iron deposition was related to some clinical characteristics, such as the recurrence rate and duration disease.

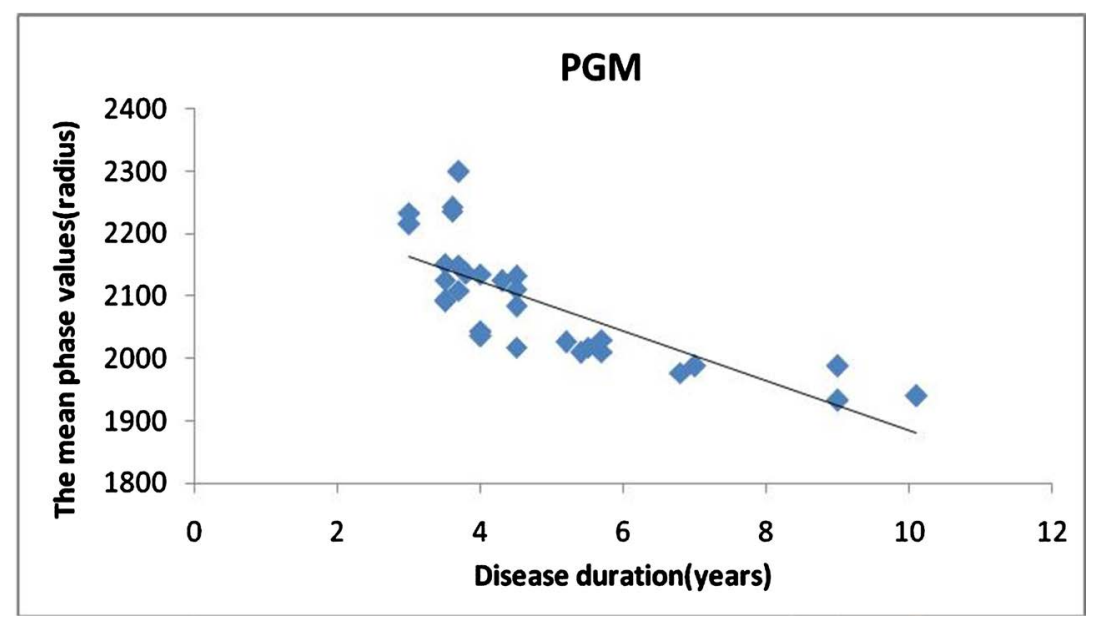

Figure 3. Correlation between mean phase value in the precentral gyrus and disease duration for the RRMS patients $\left(r_{s}=-0.399, P<0.05\right)$. 
The preliminary results of our team showed that RRMS patients had increased iron deposition in the PGM, compared with the normal control group [10]. And this study got the same results. We were also continuing to focus on the longitudinal studying the corrlelation between the iron deposition in the PGM and the volume of the precentral gyrus, in order to understand the pathophysiologic basis for iron deposition in RRMS patients. We investigated that the MPVs in the PGM in RRMS patients decreased during the period, that is, iron deposition increased with the progression of disease. Recently, more attention has been paid to the role of iron in the pathophysiology of MS. However, the mechanisms of iron accumulation in multiple sclerosis are incompletely understood. Potential mechanisms include abnormal iron deposits can occur as extracellular deposits or as extravasated red blood cells and their breakdown products. In addition, an abnormally high iron concentration has been detected in mitochondria, microglia, macrophages, neurons, and along vessels. And altered neurotransmitter metabolism of dopamine or glutamate; activation of $\mathrm{N}$-methyl-D-aspartate receptor, which could enhance iron uptake; or altered local energy demands [11].

In addition, we demonstated that the MPVs in the PGM were positively correlated with the volume of the precentral gyrus, that is to say, the iron deposition is increasing, but the volume of the precentral gyrus is atrophy. The possible reason is that which may be due to the generation of free radicals induced by the increased iron level, the lipid peroxidation, the degeneration and loss of neurons, which lead to the brain atrophy [12]. However, the recent study [12] was to measure the magnetic susceptibility with 3.0T MRI for 600 participants with MS (452 with RRMS and 148 with second progressive MS) and 250 age- and sex-matched healthy control participants, it showed that altered deep gray matter iron is associated with the evolution of MS and on disability accrual, independent of tissue atrophy. So the relationship between the iron deposition and the brain atrophy is need to further study in the future.

Interestingly, longitudinal comparison of the two examinations in RRMS showed that the MPV of the precentral gyrus gray matter decreased, while the volume of the precentral gyrus showed no significant change. Therefore, it was speculated that the iron deposition might precede the brain tissue atrophy, or the iron deposition developed faster, while the volume change of the precentral gyrus was relatively slow. The two progresses were inconsistent, which was similar to the results of Hagemeier et al. [13]. They also measured RRMS patients and $61 \mathrm{Sec}-$ ondary Progressive Multiple Sclerosis (SPMS) patients of the average value and phase volume in deep brain gray matter nuclei [7], it is concluded that iron content increased and atrophy in deep brain gray matter nuclei, also concluded that iron deposition and atrophy in deep brain gray matter nuclei are more powerful influence than lesion load of white matter and gray matter atrophy lead to disability MS patients. The other study [14] evaluated ninety-five RRMS patients iron deposition and thalamus volume included quantitative susceptibility mapping and 3D $\mathrm{T}_{1}$ echo-spoiled gradient-echo sequences underwent 3T MR imag- 
ing. They also assessed associations between thalamus susceptibility and total gray matter volume. It showed that iron levels in the thalamus are associated with $\mathrm{T}_{2}$ lesion burden and the presence of enhancing lesions, but not with thalamus or gray matter volumes, suggesting that iron accumulation is associated with white matter inflammation.

In addition, we found that the positive correlation between duration disease and iron deposition in the precentral gray matter, That is to say, as the disease progresses, the more iron deposition in the PGM in RRMS patients; the same results with Zivadinov et al. [15]. They compared 169 cases of RRMS, 126 cases of normal control group and 64 cases of SPMS, found that the phase value increased significantly using SWI in MS patients, and have obvious correlation with lesion load. The possible reason is that with the increase of the lesion load, progressive demyelination aggravation, blocked the iron transport path lead to increase of iron deposition in gray matter. Another reason is that it may be inflammation is aggravating, the blood-brain barrier damage, extravasation of red blood cells number and area increase, cracking is associated with increased hemosiderin deposition [16] [17]. We also found that significant negative correlations were observed between the recurrence rate and the change of MPVs. Recent literatures [18] have reported that meningeal inflammation is related to the lesion of gray matter. While the precentral gyrus is adjacent to the meninges, which can be complicated with meningeal inflammation when recurrence, thus aggravating the deposition of iron on the surface of the precentral gyrus gray matter, but the specific mechanism needs further study.

We showed that the difference between the EDSS score and the volume of the precentral gyrus was negatively correlation. Because the EDSS score was mainly related to the motor function disability of RRMS patients, and the precentral gyrus was the mainly governs body movement functional area. Lansley et al. [19] used voxel-based Morphometry (VBM) to find that EDSS score was significantly negatively correlated with left precentral gyrus and posterior central gyrus atrophy, and the result is in accordance with the study. However, there was no correlation between the EDSS scores and the change of iron content in the precentral gray matter. The possible reasons are as follows: firstly, the time for patients to take examination after the onset of the disease is inconsistent, and the speed of disease progression is also inconsistent. Secondly, some patients may have received clinical treatment before the examination, which may affect the reliability of EDSS score to some extent.

There were some limitations to our work that should be mentioned. Firstly, our results should be interpreted with caution because of the small sample size, which may not be an accurate reflection of the changes in iron in the gray matter of MS patients. Secondly, this study was that the follow-up interval was short. Thirdly, Thought iron content can be quantitatively measured more easily and more accurately with ESWAN compared to $\mathrm{T}_{2}{ }^{*}$. But 3D-ESWAN is a MRI method for measuring iron content in the gray matter. Fourthly, we did not take the age into account in the precentral gyrus gray matter. Finally, The Precentral Gyrus 
GM volumes were calculated only five sections by using Image $J$ image analysis software, do not incorporate whole volume, which may have had some impact on our results. In the future, we will take more factors into account in order to assess the iron deposition accurately in RRMS patients.

\section{Conclusion}

In our study, there is abnormal iron deposition in the precentral gray matter. As the course of the disease progresses, the deposition of iron in the gray matter of the precentral gray matter increases, while the volume of the precentral gray matter shows no significant change in the short term, suggesting that iron deposition may precede brain atrophy, or the iron deposition developed faster than atrophy, and iron may be a marker of the progression of neurodegenerative diseases.

\section{Role of the Funding Source}

This work was supported by the Medicine Scientific Key Research Project of Chongqing Municipal Health and Family Planning Commission of China (NO. 2016ZDXM002), the Chongqing Basic Research and Frontier Exploration Project of Chongqing Science and Technology Commission (NO. cstc2018jcyjAX0584), the National Key Research and Development Plan of Ministry of Science and Technology of the People's Republic of China (NO. 2016YFC0107109), the Medical Scientific Youth Project of Chongqing Municipal Health and Family Planning Commission of China (NO. 2018QNXM004) and the cultivate fund of the First Affiliated Hospital of Chongqing Medical University (NO.PYJJ2019-213).

\section{Conflicts of Interest}

The authors declare no conflicts of interest regarding the publication of this paper.

\section{References}

[1] Lublin, F.D. and Reingold, S.C. (1996) Defining the Clinical Course of Multiple Sclerosis: Results of an International Survey. National Multiple Sclerosis Society (USA) Advisory Committee on Clinical Trials of New Agents in Multiple Sclerosis. Neurology, 46, 907-911. https://doi.org/10.1212/WNL.46.4.907

[2] Batista, S., Zivadinov, R., Hoogs, M., Bergsland, N., Heininen-Brown, M., Dwyer, M.G., et al. (2012) Basal Ganglia, Thalamus and Neocortical Atrophy Predicting Slowed Cognitive Processing in Multiple Sclerosis. Journal of Neurology, 259, 139-146. https://doi.org/10.1007/s00415-011-6147-1

[3] Neema, M., Arora, A., Brian, C., et al. (2009) Deep Gray Matter Involvement on Brain MRI Scans Is Associated with Clinical Progression in Multiple Sclerosis. Journal of Neuroimaging, 19, 3-8. https://doi.org/10.1111/j.1552-6569.2008.00296.x

[4] LeVine, S.M., Bilgen, M. and Lynch, S.G. (2013) Iron Accumulation in Multiple Sclerosis: An Early Pathogenic Event. Expert Review of Neurotherapeutics, 13, 247-250. https://doi.org/10.1586/ern.13.14 
[5] Chen, X., Zeng, C., Luo, T., Ouyang, Y., Lv, F., Rumzan, R., et al. (2012) Iron Deposition of the Deep Grey Matter in Patients with Multiple Sclerosis and Neuromyelitis Optica: A Control Quantitative Study by 3D-Enhanced Susceptibility-Weighted Angiography (ESWAN). European Journal of Radiology, 81, e633-e639. https://doi.org/10.1016/j.ejrad.2012.01.003

[6] Walsh, A.J., Blevins, G., Lebel, R.M., et al. (2014) Longitudinal MR Imaging of Iron in Multiple Sclerosis: An Imaging Marker of Disease. Radiology, 270, 186-196. https://doi.org/10.1148/radiol.13130474

[7] Hagemeier, J., Weinstock-Guttman, B., Heininen-Brown, M., et al. (2013) Gray Matter SWI-Filtered Phase and Atrophy Are Linked to Disability in MS. Frontiers in Bioscience (Elite ED), 5, 525-532. https://doi.org/10.2741/E634

[8] Du, S.L., Sah, S.K., Zeng, C., et al. (2015) Iron Deposition in the Gray Matter in Patients with Relapse-Remitting Multiple Sclerosis: A Longitudinal Study Using Three-Dimensional (3D)-Enhanced $\mathrm{T}_{2}{ }^{\star}$-Weighted Angiography (ESWAN). European Journal of Radiology, 84, 1325-1332.

https://doi.org/10.1016/j.ejrad.2015.04.013

[9] Zeng, C., Chen, X., Li, Y.M., et al. (2012) Cerebral Vein Changes in Relapsing-Remitting Multiple Sclerosis Demonstrated by Three-Dimensional Enhanced $\mathrm{T}_{2}{ }^{*}$-Weighted Angiography at 3.0 T. European Radiology, 23, 869-878. https://doi.org/10.1007/s00330-012-2637-5

[10] Rumzan, R., Wang, J.J., Zeng, C., et al. (2013) Iron Deposition in the Precentral Grey Matter in Patients with Multiple Sclerosis: A Quantitative Study Using Susceptibility-Weighted Imaging. European Journal of Radiology, 82, e95-e99. https://doi.org/10.1016/j.ejrad.2012.09.006

[11] Zivadinov, R., Weinstock-Guttman, B. and Pirko, I. (2011) Iron Deposition and Inflammation in Multiple Sclerosis. Which One Comes First? BMC Neuroscience, 12, 60. https://doi.org/10.1186/1471-2202-12-60

[12] Zivadinov, R., Tavazzi, E., Bergsland, N., et al. (2018) Brain Iron at Quantitative MRI Is Associated with Disability in Multiple Sclerosis. Radiology, 289, 487-496. https://doi.org/10.1148/radiol.2018180136

[13] Hagemeier, J., Weinstock-Guttman, B., Bergsland, N., et al. (2012) Iron Deposition on SWI-Filtered Phase in the Subcortical Deep Gray Matter of Patients with Clinically Isolated Syndrome May Precede Structure-Specific Atrophy. AJNR, 33, 1596-1601. https://doi.org/10.3174/ajnr.A3030

[14] Chiang, G.C., Hu, J. and Morris, E. (2018) Quantitative Susceptibility Mapping of the Thalamus: Relationships with Thalamic Volume, Total Gray Matter Volume, and T2 Lesion Burden. American Journal of Neuroradiology, 39, 467-472. https://doi.org/10.3174/ajnr.A5537

[15] Zivadinov, R., Heininen-Brown, M., Schirda, C.V., et al. (2012) Abnormal Subcortical Deep-Gray Matter Susceptibility-Weighted Imaging Filtered Phase Measurements in Patients with Multiple Sclerosis: A Case-Control Study. Neuroimage, 59, 331-339. https://doi.org/10.1016/j.neuroimage.2011.07.045

[16] Singh, A.V. and Zamboni, P. (2009) Anomalous Venous Blood Flow and Iron Deposition in Multiple Sclerosis. Journal of Cerebral Blood Flow \& Metabolism, 29, 1867-1878. https://doi.org/10.1038/jcbfm.2009.180

[17] Ge, Y., Jensen, J.H., et al. (2007) Quantitative Assessment of Iron Accumulation in the Deep Gray Matter of Multiple Sclerosis by Magnetic Field Correlation Imaging. American Journal of Neuroradiology, 28, 1639-1644. https://doi.org/10.3174/ajnr.A0646 
[18] Bevan, R.J., Evans, R., Griffiths, L., et al. (2018) Meningeal Inflammation and Cortical Demyelination in Acute Multiple Sclerosis. Annals of Neurology, 84, 829-842. https://doi.org/10.1002/ana.25365

[19] Lansley, J., Mataix-cols, D., Grau, M., et al. (2013) Localized Grey Matter Atrophy in Multiple Sclerosis: A Meta-Analysis of Voxel-Based Morphometry Studies and Associations with Functional Disability. Neuroscience \& Biobehavioral Reviews, 37, 819-830. https://doi.org/10.1016/j.neubiorev.2013.03.006 